\title{
THE COMPARISON OF RESULTS OBTAINED FROM THE CONTINUOUS AND DISCONTINUOUS GALERKIN METHOD FOR THE THERMOELASTICITY PROBLEM
}

\author{
Ewa Wegrzyn-Skrzypczak \\ Department of Mathematics, Czestochowa University of Technology \\ Częstochowa, Poland \\ ewa.skrzypczak@im.pcz.pl
}

Received: 10 February 2021; Accepted: 23 March 2021

\begin{abstract}
The presented paper is focused on the comparison of the Continuous and Discontinuous Galerkin Methods in terms of thermoelasticity for a cubic element. For this purpose, a numerical model of the phenomenon was built using both methods together with the Finite Element Method (FEM). The comparison of the results of numerical simulation obtained with the use of an original computer program based on the derived final set of FEM equations for both methods is presented.
\end{abstract}

MSC 2010: 35J25, 65M60

Keywords: thermoelasticity, Finite Element Method, discontinuous Galerkin method

\section{Introduction}

Mechanical and thermal loads as well as the type of used supports are the most important factors that determine the behaviour of the each construction element [1]. The procedure for determining stresses caused by thermal load using the Continuous Galerkin Method (CGM), i.e. the classical approach to the Finite Element Method (FEM), has been discussed in details in [2]. The use of CGM entails the necessity of treating the analyzed area in a global way, building a huge stiffness matrix (especially in 3D cases) and solving a large number of linear equations. The application of the Discontinuous Galerkin method (DGM) [3,4] to solve the spatial problem of thermoelasticity has been discussed in [5].

Comparing these two methods, it can be stated that the differences between them are quite significant. They mainly concern the way of writing the basic equations and the methodology of deriving their discrete form. CGM works well for elliptic differential equations and steady processes, while DGM is better used for hyperbolic equations and transient processes [6]. The problem of thermoelasticity belongs to the steady processes and, in addition, the equations describing problem include second-order derivatives, which is a significant problem for DGM which has an impact on the effectiveness of the method in terms of computation time. 
Based on assumptions of the mathematical and numerical models presented in $[2,5]$, two proprietary solvers were prepared. Both solvers can be used in numerical calculations of coupled physical phenomena. The comparison of the obtained results make it possible to determine the effectiveness of both methods for the considered problem.

\section{Mathematical and numerical description}

The problem of determining elastic deformations in the three-dimensional region $\Omega$ (Fig. 1) is based on the known displacements $u_{x}(x, y, z), u_{y}(x, y, z)$, $u_{z}(x, y, z)$ on the surfaces $\partial \Omega_{1}, \partial \Omega_{2}, \partial \Omega_{3}$ and the temperature field $T(x, y, z)$.

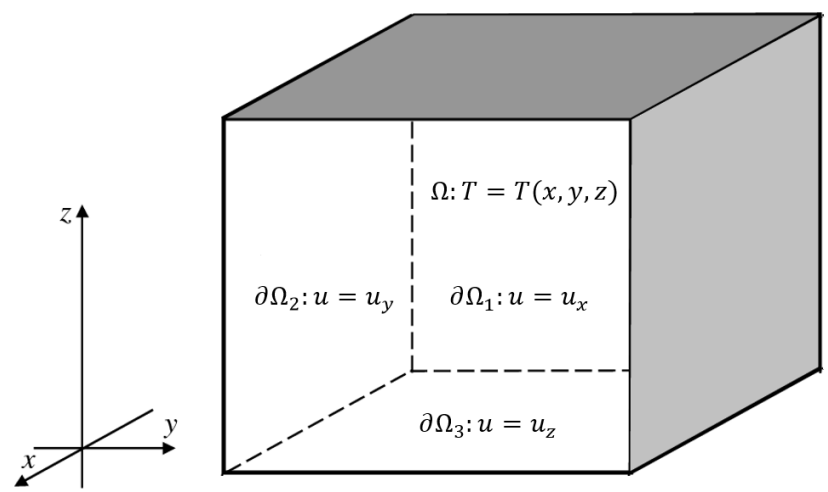

Fig. 1. Considered volume $\Omega$ with the temperature field $T(x, y, z)$ and displacements $u(x, y, z)$ on the surfaces $\partial \Omega_{1}, \partial \Omega_{2}, \partial \Omega_{3}$

In order to solve the problem at the beginning, the system of equilibrium equations (1) is considered:

$$
\begin{gathered}
\frac{\partial \sigma_{x}}{\partial x}+\frac{\partial \tau_{x y}}{\partial y}+\frac{\partial \tau_{x z}}{\partial z}+F_{x}=0 \quad \frac{\partial \tau_{x y}}{\partial x}+\frac{\partial \sigma_{y}}{\partial y}+\frac{\partial \tau_{y z}}{\partial z}+F_{y}=0 \\
\frac{\partial \tau_{x z}}{\partial x}+\frac{\partial \tau_{y z}}{\partial y}+\frac{\partial \sigma_{z}}{\partial z}+F_{z}=0
\end{gathered}
$$

where $\sigma_{x}, \sigma_{y}, \sigma_{z}$ are normal stresses, $\tau_{x y}=\tau_{y x}, \tau_{x z}=\tau_{z x}, \tau_{y z}=\tau_{z y}$ are shear stresses, $F_{x}, F_{y}, F_{z}$ are components of the body forces vector, which in the further consideration are neglected.

The starting point of the numerical consideration is the criterion of the weighted residual $[7,8]$, which was used to solve the system of equation (1). The differential equations (1) were multiplied by the weight function $w$ and integrated over the considered domain $\Omega$, assuming that the integrals equals zero: 


$$
\begin{gathered}
\int_{\Omega} w\left(\frac{\partial \sigma_{x}}{\partial x}+\frac{\partial \tau_{x y}}{\partial y}+\frac{\partial \tau_{x z}}{\partial z}\right) d \Omega=0 \int_{\Omega} w\left(\frac{\partial \tau_{x y}}{\partial x}+\frac{\partial \sigma_{y}}{\partial y}+\frac{\partial \tau_{y z}}{\partial z}\right) d \Omega=0 \\
\int_{\Omega} w\left(\frac{\partial \tau_{x z}}{\partial x}+\frac{\partial \tau_{y z}}{\partial y}+\frac{\partial \sigma_{z}}{\partial z}\right) d \Omega=0
\end{gathered}
$$

The use of boundary conditions in CGM and DGM requires the transformation of the equations (1) into the form based on displacements. Therefore, the following relationships between stresses and displacements are used:

$$
\begin{array}{ll}
\sigma_{x}=f_{1} \frac{\partial u_{x}}{\partial x}+f_{2} \frac{\partial u_{y}}{\partial y}+f_{2} \frac{\partial u_{z}}{\partial z}-f_{4} \alpha \Delta T, & \tau_{x y}=\tau_{y x}=f_{3} \frac{\partial u_{x}}{\partial y}+f_{3} \frac{\partial u_{y}}{\partial x} \\
\sigma_{y}=f_{2} \frac{\partial u_{x}}{\partial x}+f_{1} \frac{\partial u_{y}}{\partial y}+f_{2} \frac{\partial u_{z}}{\partial z}-f_{4} \alpha \Delta T, & \tau_{y z}=\tau_{z y}=f_{3} \frac{\partial u_{y}}{\partial z}+f_{3} \frac{\partial u_{z}}{\partial y} \\
\sigma_{z}=f_{2} \frac{\partial u_{x}}{\partial x}+f_{2} \frac{\partial u_{y}}{\partial y}+f_{1} \frac{\partial u_{z}}{\partial z}-f_{4} \alpha \Delta T, & \tau_{x z}=\tau_{z x}=f_{3} \frac{\partial u_{x}}{\partial z}+f_{3} \frac{\partial u_{z}}{\partial x}
\end{array}
$$

where $\alpha\left[\mathrm{K}^{-1}\right]$ is the linear coefficient of thermal expansion, $\Delta T$ is the difference between current and initial temperatures, and $f_{1}, f_{2}, f_{3}, f_{4}$ are the coefficients listed below:

$$
f_{1}=\frac{E(1-v)}{(1+v)(1-2 v)}, f_{2}=\frac{E v}{(1+v)(1-2 v)}, f_{3}=\frac{E}{2(1+v)}, f_{4}=\frac{E(1+v)}{(1+v)(1-2 v)}
$$

where $E\left[\mathrm{~N} / \mathrm{m}^{2}\right]$ is the Young's modulus and $v[-]$ represents Poisson's ratio.

Assume that the domain $\Omega$ consists of $N$ tetrahedrons:

$$
\Omega=\bigcup_{j=1}^{N} \Omega_{j}
$$

substituting relations (3) in the equations (1), one obtains the displacement dependent integral form of thermoelasticity equations:

$$
\begin{aligned}
& \int_{\Omega_{j}} w\left[\frac{\partial}{\partial x}\left(f_{1} \frac{\partial u_{x}}{\partial x}+f_{2} \frac{\partial u_{y}}{\partial y}+f_{2} \frac{\partial u_{z}}{\partial z}-f_{4} \alpha \Delta T\right)+f_{3} \frac{\partial}{\partial y}\left(\frac{\partial u_{x}}{\partial y}+\frac{\partial u_{y}}{\partial x}\right)+f_{3} \frac{\partial}{\partial z}\left(\frac{\partial u_{x}}{\partial z}+\frac{\partial u_{z}}{\partial x}\right)\right] d \Omega=0 \\
& \int_{\Omega_{j}} w\left[f_{3} \frac{\partial}{\partial x}\left(\frac{\partial u_{x}}{\partial y}+\frac{\partial u_{y}}{\partial x}\right)+\frac{\partial}{\partial y}\left(f_{2} \frac{\partial u_{x}}{\partial x}+f_{1} \frac{\partial u_{y}}{\partial y}+f_{2} \frac{\partial u_{z}}{\partial z}-f_{4} \alpha \Delta T\right)+f_{3} \frac{\partial}{\partial z}\left(\frac{\partial u_{y}}{\partial z}+\frac{\partial u_{z}}{\partial y}\right)\right] d \Omega=0 \\
& \int_{\Omega_{j}} w\left[f_{3} \frac{\partial}{\partial x}\left(\frac{\partial u_{x}}{\partial z}+\frac{\partial u_{z}}{\partial x}\right)+f_{3} \frac{\partial}{\partial y}\left(\frac{\partial u_{y}}{\partial z}+\frac{\partial u_{z}}{\partial y}\right)+\frac{\partial}{\partial z}\left(f_{2} \frac{\partial u_{x}}{\partial x}+f_{2} \frac{\partial u_{y}}{\partial y}+f_{1} \frac{\partial u_{z}}{\partial z}-f_{4} \alpha \Delta T\right)\right] d \Omega=0
\end{aligned}
$$


Details of the mathematical description of both CG and DG methods are presented in the works $[2,5]$. This paper presents only the final equations resulting from the application of FEM.

The global system of FEM equations for CGM is presented below:

$$
\mathbf{K} \cdot \mathbf{u}=\mathbf{B}
$$

where $\mathbf{K}$ is the stiffness matrix, $\mathbf{u}$ is the sought displacement vector, $\mathbf{B}$ is the right side vector. To obtain the global matrix $\mathbf{K}$ and global vector $\mathbf{B}$, one needs to sum up the $N$ local stiffness matrices $\mathbf{K}^{(e)}$ and local right side vectors $\mathbf{B}^{(e)}$ as follows:

$$
\mathbf{K}=\sum_{i=0}^{N-1} \mathbf{K}^{(i)}, \quad \mathbf{B}=\sum_{i=0}^{N-1} \mathbf{B}^{(i)}
$$

Assuming that weighting functions $w$ are the same as the shape functions $\phi$ (the Galerkin formulation) the elements of $\mathbf{K}^{(e)}$ can be written as:

$$
\begin{aligned}
& K_{(n, m)}^{(e)}=\int_{\Omega^{(e)}}\left[f_{1} \frac{\partial \phi_{n}}{\partial x} \frac{\partial \phi_{m}}{\partial x}+f_{3}\left(\frac{\partial \phi_{n}}{\partial y} \frac{\partial \phi_{m}}{\partial y}+\frac{\partial \phi_{n}}{\partial z} \frac{\partial \phi_{m}}{\partial z}\right)\right] d \Omega^{(e)} \\
& K_{(n, m+M)}^{(e)}=\int_{\Omega^{(e)}}\left(f_{2} \frac{\partial \phi_{n}}{\partial x} \frac{\partial \phi_{m}}{\partial y}+f_{3} \frac{\partial \phi_{n}}{\partial y} \frac{\partial \phi_{m}}{\partial x}\right) d \Omega^{(e)} \\
& K_{(n, m+2 M)}^{(e)}=\int_{\Omega^{(e)}}\left(f_{2} \frac{\partial \phi_{n}}{\partial x} \frac{\partial \phi_{m}}{\partial z}+f_{3} \frac{\partial \phi_{n}}{\partial z} \frac{\partial \phi_{m}}{\partial x}\right) d \Omega^{(e)} \\
& K_{(n+M, m)}^{(e)}=\int_{\Omega^{(e)}}\left(f_{2} \frac{\partial \phi_{n}}{\partial y} \frac{\partial \phi_{m}}{\partial x}+f_{3} \frac{\partial \phi_{n}}{\partial x} \frac{\partial \phi_{m}}{\partial y}\right) d \Omega^{(e)} \\
& K_{(n+M, m+M)}^{(e)}=\int_{\Omega^{(e)}}\left[f_{1} \frac{\partial \phi_{n}}{\partial y} \frac{\partial \phi_{m}}{\partial y}+f_{3}\left(\frac{\partial \phi_{n}}{\partial x} \frac{\partial \phi_{m}}{\partial x}+\frac{\partial \phi_{n}}{\partial z} \frac{\partial \phi_{m}}{\partial z}\right)\right] d \Omega^{(e)} \\
& K_{(n+M, m+2 M)}^{(e)}=\int_{\Omega^{(e)}}\left(f_{2} \frac{\partial \phi_{n}}{\partial y} \frac{\partial \phi_{m}}{\partial z}+f_{3} \frac{\partial \phi_{n}}{\partial z} \frac{\partial \phi_{m}}{\partial y}\right) d \Omega^{(e)} \\
& K_{(n+2 M, m)}^{(e)}=\int_{\Omega^{(e)}}\left(f_{2} \frac{\partial \phi_{n}}{\partial z} \frac{\partial \phi_{m}}{\partial x}+f_{3} \frac{\partial \phi_{n}}{\partial x} \frac{\partial \phi_{m}}{\partial z}\right) d \Omega^{(e)} \\
& K_{(n+2 M, m+M)}^{(e)}=\int_{\Omega^{(e)}}\left(f_{2} \frac{\partial \phi_{n}}{\partial z} \frac{\partial \phi_{m}}{\partial y}+f_{3} \frac{\partial \phi_{n}}{\partial y} \frac{\partial \phi_{m}}{\partial z}\right) d \Omega^{(e)} \\
& K_{(n+2 M, m+2 M)}^{(e)}=\int_{\Omega^{(e)}}\left[f_{1} \frac{\partial \phi_{n}}{\partial z} \frac{\partial \phi_{m}}{\partial z}+f_{3}\left(\frac{\partial \phi_{n}}{\partial x} \frac{\partial \phi_{m}}{\partial x}+\frac{\partial \phi_{n}}{\partial y} \frac{\partial \phi_{m}}{\partial y}\right)\right] d \Omega^{(e)}
\end{aligned}
$$


where $n=m=0,1, \ldots, M-1$ and $M$ is a number of shape functions for the tetrahedron.

The elements of $\mathbf{B}^{(e)}$ containing thermal loads are calculated as follows:

$$
\begin{aligned}
& B_{n}^{(e)}=\alpha f_{4} \int_{\Omega^{(e)}} \frac{\partial \varphi_{n}}{\partial x} \Delta T_{n} d \Omega^{(e)} \\
& B_{n+M}^{(e)}=\alpha f_{4} \int_{\Omega^{(e)}} \frac{\partial \varphi_{n}}{\partial y} \Delta T_{n} d \Omega^{(e)} \\
& B_{n+2 M}^{(e)}=\alpha f_{4} \int_{\Omega^{(e)}} \frac{\partial \varphi_{n}}{\partial z} \Delta T_{n} d \Omega^{(e)}
\end{aligned}
$$

At this point it should be emphasized that in the case of CGM, we have global formulation of the problem, while in the case of DGM we must derive the equations for the each finite element. Therefore, for DGM, the calculation process must be done for each finite element, and the final solution is obtained after many iterations. The matrix $\mathbf{K}^{(e)}$ is built only once at the start of the process while vector $\mathbf{B}^{(e)}$ must be rebuilt in each iteration. The final equations resulting from the application of FEM for DGM has a form:

$$
\mathbf{K}^{(e)} \cdot \mathbf{x}^{(e)}=\mathbf{B}^{(e)}
$$

To build the stiffness matrices $\mathbf{K}^{(e)}$ in the form:

$$
\begin{aligned}
& \mathbf{K}^{(e)}= \\
& {\left[\begin{array}{cccccccccccc}
\mathbf{M} & \mathbf{0} & \mathbf{0} & \mathbf{0} & \mathbf{0} & \mathbf{0} & \mathbf{0} & \mathbf{0} & \mathbf{0} & \mathbf{D}_{x} & \mathbf{0} & \mathbf{0} \\
\mathbf{0} & \mathbf{M} & \mathbf{0} & \mathbf{0} & \mathbf{0} & \mathbf{0} & \mathbf{0} & \mathbf{0} & \mathbf{0} & \mathbf{D}_{y} & \mathbf{0} & \mathbf{0} \\
\mathbf{0} & \mathbf{0} & \mathbf{M} & \mathbf{0} & \mathbf{0} & \mathbf{0} & \mathbf{0} & \mathbf{0} & \mathbf{0} & \mathbf{D}_{z} & \mathbf{0} & \mathbf{0} \\
\mathbf{0} & \mathbf{0} & \mathbf{0} & \mathbf{M} & \mathbf{0} & \mathbf{0} & \mathbf{0} & \mathbf{0} & \mathbf{0} & \mathbf{0} & \mathbf{D}_{x} & \mathbf{0} \\
\mathbf{0} & \mathbf{0} & \mathbf{0} & \mathbf{0} & \mathbf{M} & \mathbf{0} & \mathbf{0} & \mathbf{0} & \mathbf{0} & \mathbf{0} & \mathbf{D}_{y} & \mathbf{0} \\
\mathbf{0} & \mathbf{0} & \mathbf{0} & \mathbf{0} & \mathbf{0} & \mathbf{M} & \mathbf{0} & \mathbf{0} & \mathbf{0} & \mathbf{0} & \mathbf{D}_{z} & \mathbf{0} \\
\mathbf{0} & \mathbf{0} & \mathbf{0} & \mathbf{0} & \mathbf{0} & \mathbf{0} & \mathbf{M} & \mathbf{0} & \mathbf{0} & \mathbf{0} & \mathbf{0} & \mathbf{D}_{x} \\
\mathbf{0} & \mathbf{0} & \mathbf{0} & \mathbf{0} & \mathbf{0} & \mathbf{0} & \mathbf{0} & \mathbf{M} & \mathbf{0} & \mathbf{0} & \mathbf{0} & \mathbf{D}_{y} \\
\mathbf{0} & \mathbf{0} & \mathbf{0} & \mathbf{0} & \mathbf{0} & \mathbf{0} & \mathbf{0} & \mathbf{0} & \mathbf{M} & \mathbf{0} & \mathbf{0} & \mathbf{D}_{z} \\
-f_{1} \mathbf{D}_{x} & -f_{3} \mathbf{D}_{y} & -f_{3} \mathbf{D}_{z} & -f_{3} \mathbf{D}_{y} & -f_{2} \mathbf{D}_{x} & \mathbf{0} & -f_{3} \mathbf{D}_{z} & \mathbf{0} & -f_{2} \mathbf{D}_{x} & -\mathbf{S}_{x x} & -\mathbf{S}_{x y} & -\mathbf{S}_{x z} \\
-f_{2} \mathbf{D}_{y} & -f_{3} \mathbf{D}_{x} & \mathbf{0} & -f_{3} \mathbf{D}_{x} & -f_{1} \mathbf{D}_{y} & -f_{3} \mathbf{D}_{z} & \mathbf{0} & -f_{3} \mathbf{D}_{z} & -f_{2} \mathbf{D}_{y} & -\mathbf{S}_{x y} & -\mathbf{S}_{y y} & -\mathbf{S}_{y z} \\
-f_{2} \mathbf{D}_{z} & \mathbf{0} & -f_{3} \mathbf{D}_{x} & \mathbf{0} & -f_{2} \mathbf{D}_{z} & -f_{3} \mathbf{D}_{y} & -f_{3} \mathbf{D}_{x} & -f_{3} \mathbf{D}_{y} & -f_{1} \mathbf{D}_{z} & -\mathbf{S}_{x z} & -\mathbf{S}_{y z} & -\mathbf{S}_{z z}
\end{array}\right]}
\end{aligned}
$$


the following matrices and vectors are used:

$$
\begin{gathered}
\mathbf{D}_{x}=\int_{\Omega^{(e)}}\left(\frac{\partial \boldsymbol{\Phi}}{\partial x}\right) \boldsymbol{\Phi}^{T} d \Omega-\mathbf{B}_{x}, \quad \mathbf{B}_{x}=\frac{1}{2} n_{x} \int_{\partial \boldsymbol{\Omega}^{(e)}} \boldsymbol{\Phi} \boldsymbol{\Phi}^{T} d s \\
\mathbf{D}_{y}=\int_{\Omega^{(e)}}\left(\frac{\partial \boldsymbol{\Phi}}{\partial y}\right) \boldsymbol{\Phi}^{T} d \Omega-\mathbf{B}_{y}, \quad \mathbf{B}_{y}=\frac{1}{2} n_{y} \int_{\partial \Omega^{(e)}} \boldsymbol{\Phi} \boldsymbol{\Phi}^{T} d s \\
\mathbf{D}_{z}=\int_{\Omega^{(e)}}\left(\frac{\partial \boldsymbol{\Phi}}{\partial x}\right) \boldsymbol{\Phi}^{T} d \Omega-\mathbf{B}_{z}, \quad \mathbf{B}_{z}=\frac{1}{2} n_{z} \int_{\partial \Omega^{(e)}} \boldsymbol{\Phi} \boldsymbol{\Phi}^{T} d s \\
\mathbf{S}_{x x}^{\left(u_{x}\right)}=C_{11}\left(f_{1} n_{x}^{2}+f_{3} n_{y}^{2}+f_{3} n_{z}^{2}\right) \int_{\partial \Omega_{j}} \boldsymbol{\Phi} \boldsymbol{\Phi}^{T} d s \quad \mathbf{S}_{x y}^{\left(u_{y}\right)}=C_{11}\left(f_{3}+f_{2}\right) n_{y} n_{x} \int_{\partial \Omega_{j}} \boldsymbol{\Phi} \boldsymbol{\Phi}^{T} d s \\
\mathbf{S}_{y y}^{\left(u_{y}\right)}=C_{11}\left(f_{3} n_{x}^{2}+f_{1} n_{y}^{2}+f_{3} n_{z}^{2}\right) \int_{\partial \Omega_{j}} \boldsymbol{\Phi} \boldsymbol{\Phi}^{T} d s \quad \mathbf{S}_{x z}^{\left(u_{z}\right)}=C_{11}\left(f_{3}+f_{2}\right) n_{z} n_{x} \int_{\partial \Omega_{j}} \boldsymbol{\Phi} \boldsymbol{\Phi}^{T} d s \\
\mathbf{S}_{z z}^{\left(u_{z}\right)}=C_{11}\left(f_{3} n_{x}^{2}+f_{3} n_{y}^{2}+f_{1} n_{z}^{2}\right) \int_{\partial \Omega_{j}} \boldsymbol{\Phi} \boldsymbol{\Phi}^{T} d s \quad \mathbf{S}_{y z}^{\left(u_{z}\right)}=C_{11}\left(f_{3}+f_{2}\right) n_{z} n_{y} \int_{\partial \Omega_{j}}^{\mathbf{\Phi} \boldsymbol{\Phi}^{T} d s} \\
\mathbf{M}=\int_{\Omega^{(e)}} \boldsymbol{\Phi} \boldsymbol{\Phi}^{T} d \Omega
\end{gathered}
$$

where $\boldsymbol{\Phi}$ is a vector of shape functions, $C_{11}$ is a constant providing convergence of the solution whose value is chosen experimentally, $n_{x}, n_{y}, n_{z}$ are the components of the vector perpendicular to the walls of the finite element.

The sought displacement vector $\mathbf{x}^{(e)}$ can be written in the form:

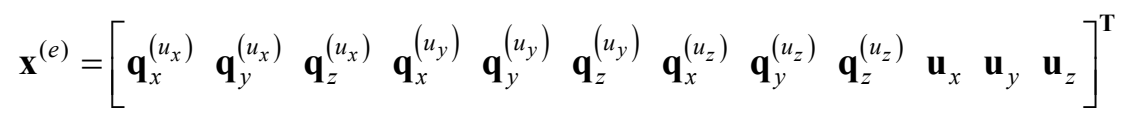

where $\mathbf{q}$ are vectors of additional variables introduced instead of derived displacements to the lower order of the equation, $\mathbf{u}$ are vectors of displacement.

One can write the local right side vectors $\mathbf{B}^{(e)}$ as:

$$
\mathbf{B}^{(e)}=\mathbf{F}^{(e)} \cdot \mathbf{y}^{(e)}+\mathbf{H}^{(e)}
$$

where

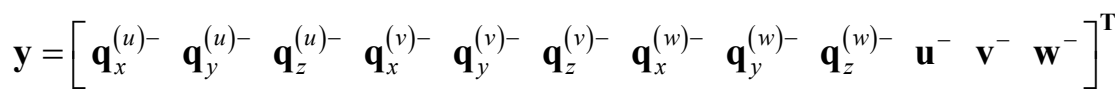




$\mathbf{F}^{(e)}=\left[\begin{array}{cccccccccccc}\mathbf{0} & \mathbf{0} & \mathbf{0} & \mathbf{0} & \mathbf{0} & \mathbf{0} & \mathbf{0} & \mathbf{0} & \mathbf{0} & \mathbf{B}_{x} & \mathbf{0} & \mathbf{0} \\ \mathbf{0} & \mathbf{0} & \mathbf{0} & \mathbf{0} & \mathbf{0} & \mathbf{0} & \mathbf{0} & \mathbf{0} & \mathbf{0} & \mathbf{B}_{y} & \mathbf{0} & \mathbf{0} \\ \mathbf{0} & \mathbf{0} & \mathbf{0} & \mathbf{0} & \mathbf{0} & \mathbf{0} & \mathbf{0} & \mathbf{0} & \mathbf{0} & \mathbf{B}_{z} & \mathbf{0} & \mathbf{0} \\ \mathbf{0} & \mathbf{0} & \mathbf{0} & \mathbf{0} & \mathbf{0} & \mathbf{0} & \mathbf{0} & \mathbf{0} & \mathbf{0} & \mathbf{0} & \mathbf{B}_{x} & \mathbf{0} \\ \mathbf{0} & \mathbf{0} & \mathbf{0} & \mathbf{0} & \mathbf{0} & \mathbf{0} & \mathbf{0} & \mathbf{0} & \mathbf{0} & \mathbf{0} & \mathbf{B}_{y} & \mathbf{0} \\ \mathbf{0} & \mathbf{0} & \mathbf{0} & \mathbf{0} & \mathbf{0} & \mathbf{0} & \mathbf{0} & \mathbf{0} & \mathbf{0} & \mathbf{0} & \mathbf{B}_{z} & \mathbf{0} \\ \mathbf{0} & \mathbf{0} & \mathbf{0} & \mathbf{0} & \mathbf{0} & \mathbf{0} & \mathbf{0} & \mathbf{0} & \mathbf{0} & \mathbf{0} & \mathbf{0} & \mathbf{B}_{x} \\ \mathbf{0} & \mathbf{0} & \mathbf{0} & \mathbf{0} & \mathbf{0} & \mathbf{0} & \mathbf{0} & \mathbf{0} & \mathbf{0} & \mathbf{0} & \mathbf{0} & \mathbf{B}_{y} \\ \mathbf{0} & \mathbf{0} & \mathbf{0} & \mathbf{0} & \mathbf{0} & \mathbf{0} & \mathbf{0} & \mathbf{0} & \mathbf{0} & \mathbf{0} & \mathbf{0} & \mathbf{B}_{z} \\ -f_{1} \mathbf{B}_{x} & -f_{3} \mathbf{B}_{y} & -f_{3} \mathbf{B}_{z} & -f_{3} \mathbf{B}_{y} & -f_{2} \mathbf{B}_{x} & \mathbf{0} & -f_{3} \mathbf{B}_{z} & \mathbf{0} & -f_{2} \mathbf{B}_{x} & -\mathbf{S}_{x x} & -\mathbf{S}_{x y} & -\mathbf{S}_{x z} \\ -f_{2} \mathbf{B}_{y} & -f_{3} \mathbf{B}_{x} & \mathbf{0} & -f_{3} \mathbf{B}_{x} & -f_{1} \mathbf{B}_{y} & -f_{3} \mathbf{B}_{z} & \mathbf{0} & -f_{3} \mathbf{B}_{z} & -f_{2} \mathbf{B}_{y} & -\mathbf{S}_{x y} & -\mathbf{S}_{y y} & -\mathbf{S}_{y z} \\ -f_{2} \mathbf{B}_{z} & \mathbf{0} & -f_{3} \mathbf{B}_{x} & \mathbf{0} & -f_{2} \mathbf{B}_{z} & -f_{3} \mathbf{B}_{y} & -f_{3} \mathbf{B}_{x} & -f_{3} \mathbf{B}_{y} & -f_{1} \mathbf{B}_{z} & -\mathbf{S}_{x z} & -\mathbf{S}_{y z} & -\mathbf{S}_{z z}\end{array}\right]$

$$
\begin{gathered}
\mathbf{H}^{(e)}=\left[\begin{array}{llllllllllll}
0 & 0 & 0 & 0 & 0 & 0 & 0 & 0 & 0 & \mathbf{H}_{x} & \mathbf{H}_{y} & \mathbf{H}_{z}
\end{array}\right]^{\mathbf{T}} \\
\mathbf{H}_{x}=-f_{4} \alpha \int_{\Omega^{(e)}}\left(\frac{\partial \boldsymbol{\Phi}}{\partial x}\right) \boldsymbol{\Phi}^{T} \Delta \mathbf{T} d V-\mathbf{h}_{x}, \quad \mathbf{h}_{x}=\frac{1}{2} f_{4} \alpha n_{x} \int_{\partial \Omega^{(e)}} \boldsymbol{\Phi} \boldsymbol{\Phi}^{T} \Delta \mathbf{T} d s \\
\mathbf{H}_{y}=-f_{4} \alpha \int_{\Omega^{(e)}}\left(\frac{\partial \boldsymbol{\Phi}}{\partial y}\right) \boldsymbol{\Phi}^{T} \Delta \mathbf{T} d V-\mathbf{h}_{y}, \quad \mathbf{h}_{y}=\frac{1}{2} f_{4} \alpha n_{y} \int_{\partial \Omega^{(e)}} \boldsymbol{\Phi} \boldsymbol{\Phi}^{T} \Delta \mathbf{T} d s \\
\mathbf{H}_{z}=-f_{4} \alpha \int_{\Omega^{(e)}}\left(\frac{\partial \boldsymbol{\Phi}}{\partial z}\right) \boldsymbol{\Phi}^{T} \Delta \mathbf{T} d V-\mathbf{h}_{z}, \quad \mathbf{h}_{z}=\frac{1}{2} f_{4} \alpha n_{z} \int_{\partial \Omega^{(e)}} \boldsymbol{\Phi} \boldsymbol{\Phi}^{T} \Delta \mathbf{T} d s
\end{gathered}
$$

\section{Example of calculations}

The calculations were focused on determining the displacement, strain and stress fields in a steel cube, constrained in such a way as to eliminate the effect of supports (Fig. 2).

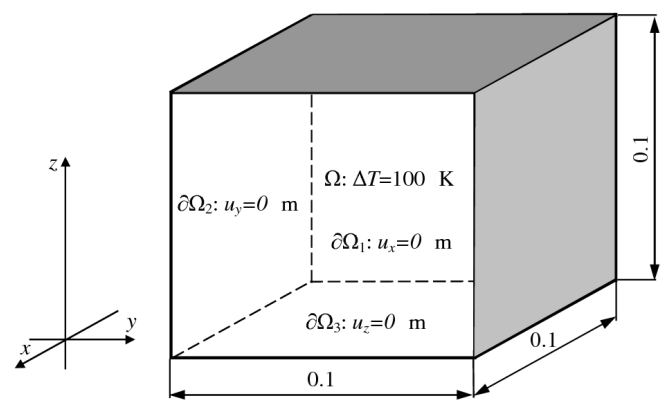

Fig. 2. Geometry of the thermally loaded cube mounted on three mutually perpendicular surfaces $\partial \Omega_{1}, \partial \Omega_{2}, \partial \Omega_{3}$ with the boundary conditions 
This type of constraints allows the heated element to expand in three directions. Only thermal deformations are non-zero, the value of which can be easily calculated. When the element is heated by $\Delta T=100 \mathrm{~K}$, the thermal deformation in each finite element is $\varepsilon_{T}=\alpha \Delta T=1.2 \cdot 10^{-5} \cdot 100=1.2 \cdot 10^{-3}$, and the corresponding displacements in each direction are the same and equal $\varepsilon_{T} L=1.2 \cdot 10^{-3} \cdot 0.1=1.2 \cdot 10^{-4} \mathrm{~m}$ on the non-constrained walls.

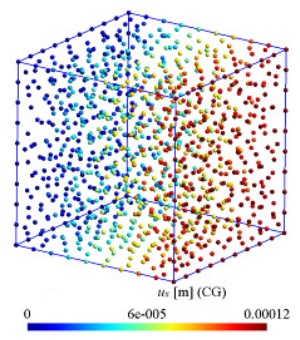

(a)

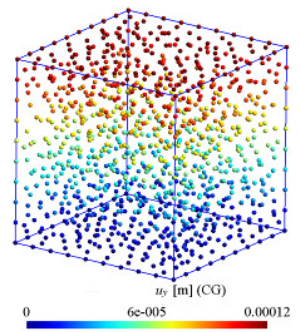

(d)

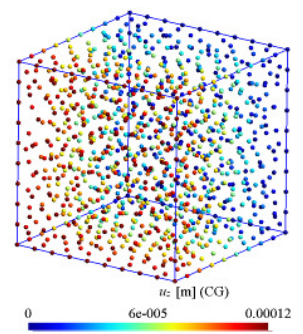

(g)

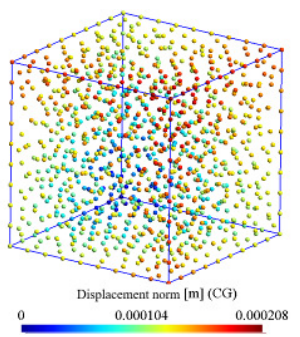

(j)

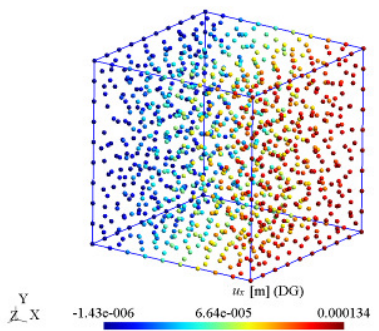

(b)

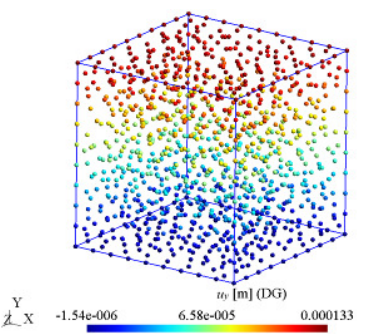

(e)

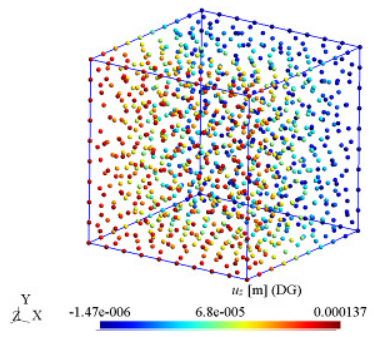

(h)

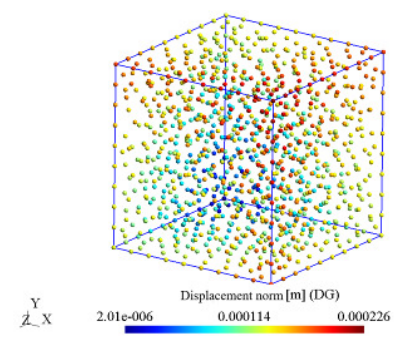

(k)

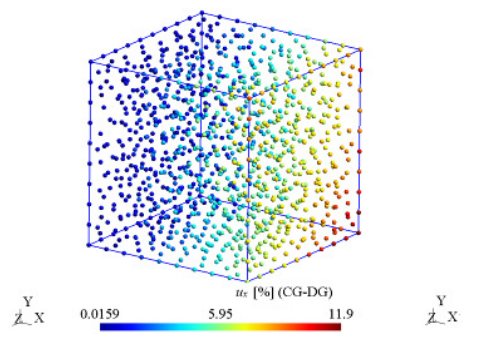

(c)

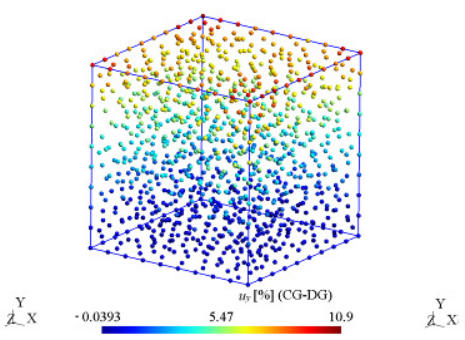

(f)

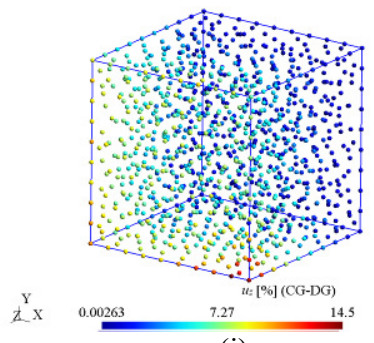

(i)

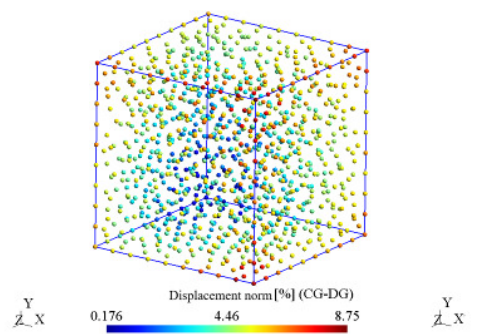

(1)

Fig. 3. Displacements $u_{x}, u_{y}, u_{z}, u$ for CG method (a, d, g, j), for DG method (b, e, h, k), percentage difference between CG and DG $(c, f, i, l)$. Results obtained for approximation order $p=1$ 
The cube was divided into the set of finite elements. Spatial discretization was carried out using the GMSH [9] open source software. The average dimension of the element was assumed as $0.01 \mathrm{~m}$, resulting in a mesh of 5057 tetrahedrons. The calculations were performed using the DGM and CGM solvers. The calculations were made for the approximation order $p=1$ and $p=2$.

The comparison of the displacements presented in Figure 3 shows clear differences between CGM and DGM in the case of linear approximation. The error calculated as the difference between the resultant displacements calculated with the use of CGM and DGM, divided by the maximum displacement in the area and multiplied by 100 , reaches $8.75 \%$, with the assumed tolerance $\varepsilon=10^{-9}$.

In the case of the approximation of the second order, the maximum error of the calculated resultant displacements decreased to $4.96 \%$. The highest error values are at the corners of the cube. Unfortunately, the reduction of error entailed a huge increase in computation time (Table 1).

Table 1. The comparison of computation time for CGM and DGM

\begin{tabular}{|c|c|c|}
\hline & CGM $\varepsilon=10^{-25}$ & DGM $\varepsilon=10^{-9}$ \\
\cline { 2 - 3 } & Computation time $[\mathrm{s}]$ & Computation time [s] \\
\hline$p=1$ & 3 & 3000 \\
\hline$p=2$ & 60 & 21600 \\
\hline
\end{tabular}

In Table 1 , the calculation time necessary to achieve the assumed precision is presented. In the case of CGM, $\varepsilon=10^{-9}$ was assumed, the computation time increased significantly with increasing the approximation order, but compared to DGM, it was definitely shorter. For $p=1$, DGM gave a convergent solution with $\varepsilon=10^{-9}$ after a time one thousand longer than CGM. In the case of $p=2$, this time was 360 times longer.

The theory shows that the elastic stresses for assumed constraints should be zero because the heated element can freely expand. Fig. 5-6 presented normal stress components calculated for both methods. The results prove that CGM complies with the theory. In this case, deviations from zero are negligible both for $p=1$ and for $p=2$. Unfortunately, DGM generates "artifacts" of incorrect stress values that occur especially frequently near the edges. For $p=2$, the erroneous values are smaller, but still significant.

In Table 2, the number of degrees of freedom in each calculation is presented. In the case of DGM, a very large number of degrees of freedom are observed, the determination of which in the computational process becomes very timeconsuming with the increase of the approximation order. 


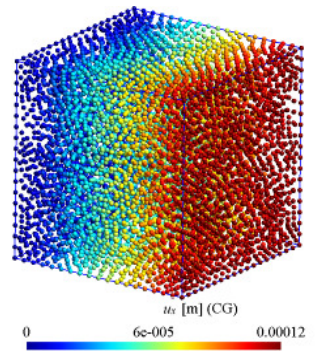

(a)

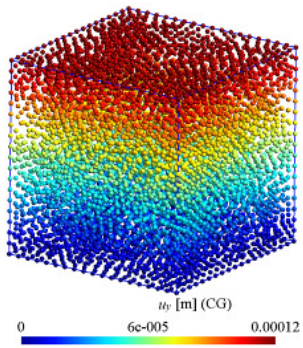

(d)

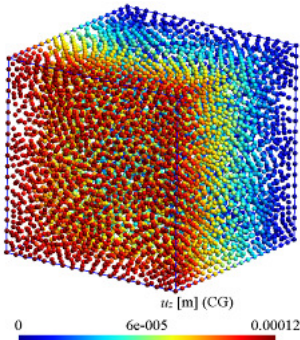

(g)

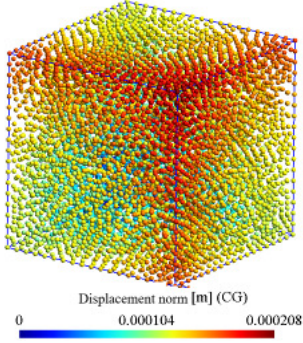

(j)

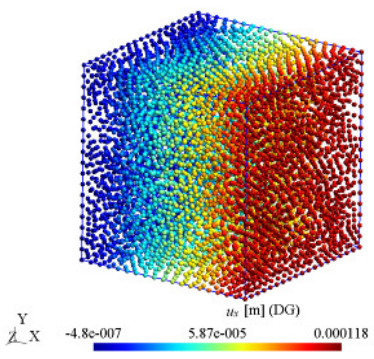

(b)

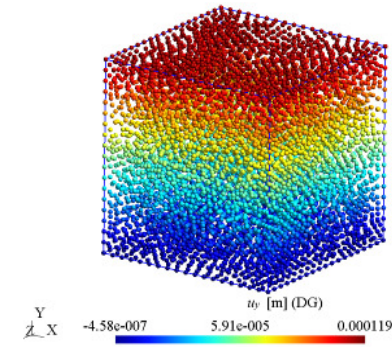

(e)

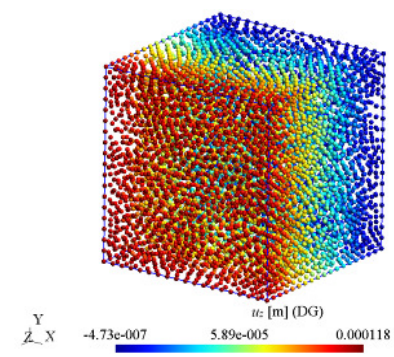

(h)

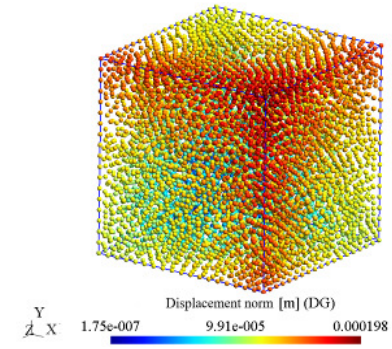

(k)

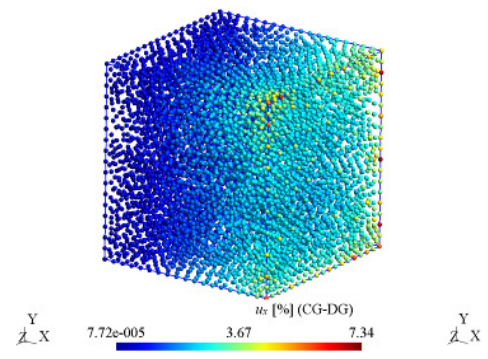

(c)

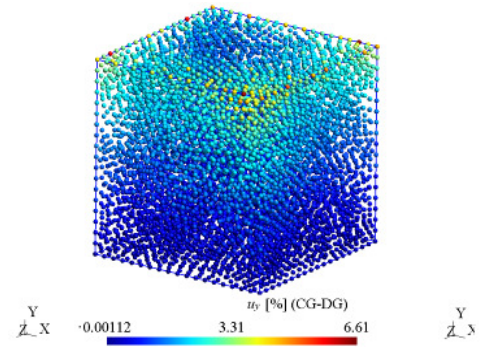

(f)

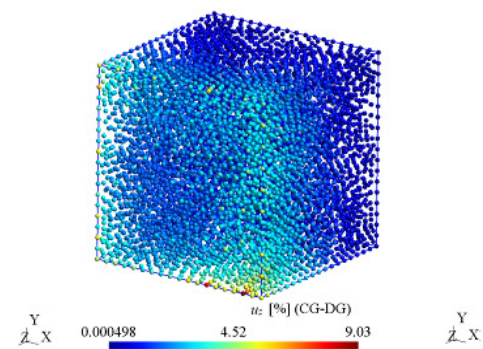

(i)

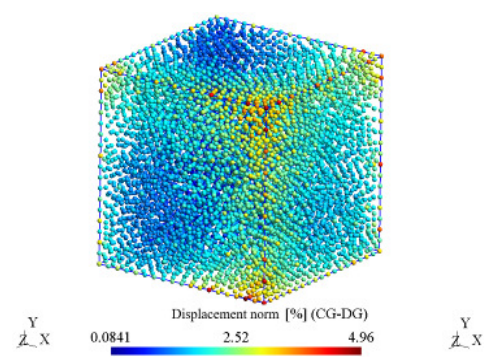

(1)

Fig. 4. Displacements $u_{x}, u_{y}, u_{z}$, $u$ for CG method (a, d, g, j), for DG method (b, e, h, k), percentage difference between CG and DG (c, f, i, l). Results obtained for approximation order $p=2$ 


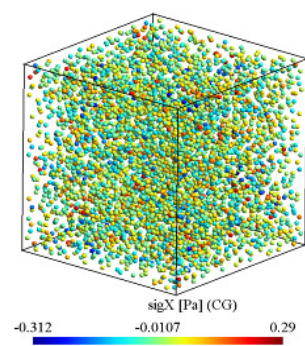

(a)

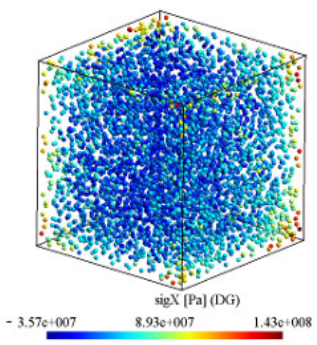

(d)

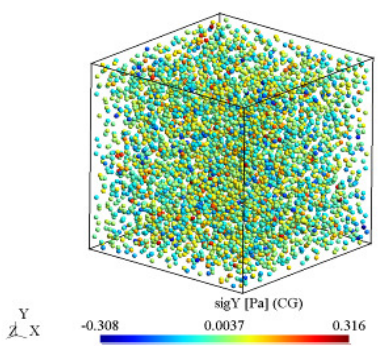

(b)

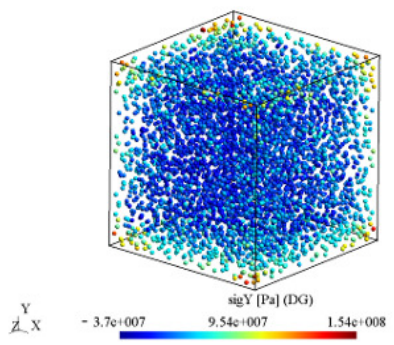

(e)

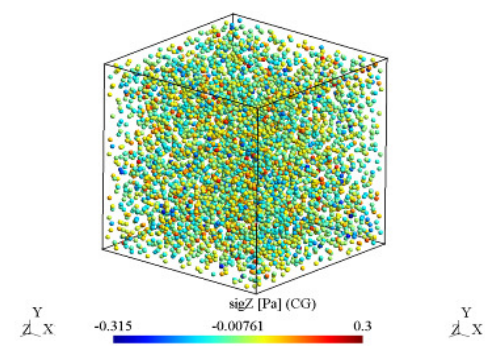

(c)

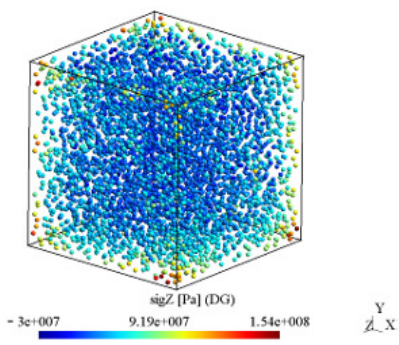

(f)

Fig. 5. Normal stress components $\sigma_{x}, \sigma_{y}, \sigma_{z}$ for CG method (a, b, c), for DG method (d, e, f). Results obtained for approximation order $p=1$

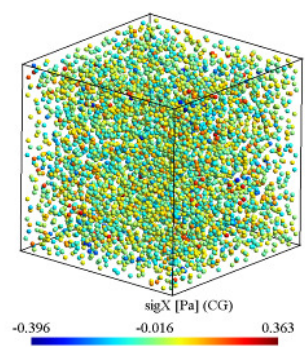

(a)

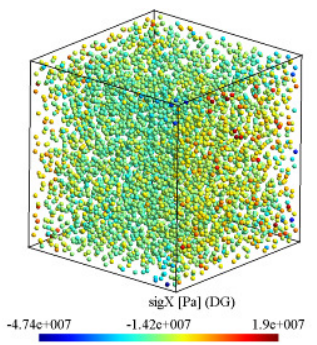

(d)

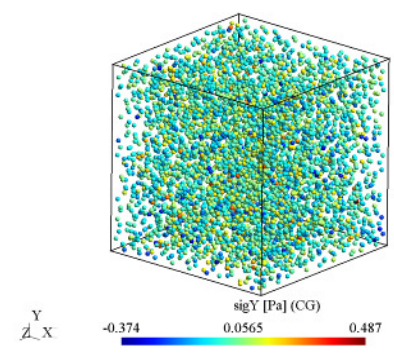

(b)

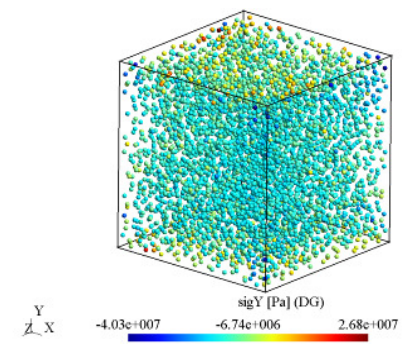

(e)

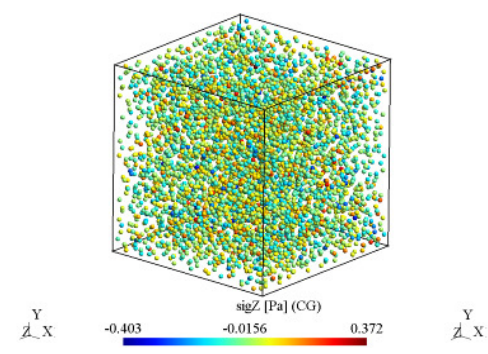

(c)

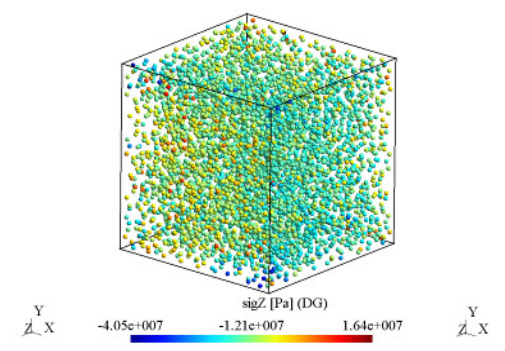

(f)

Fig. 6. Normal stress components $\sigma_{x}, \sigma_{y}, \sigma_{z}$ for CG method (a, b, c), for DG method (d, e, f). Results obtained for approximation order $p=2$ 
Table 2. Number of nodes and degrees of freedom in the test mesh for CGM and DGM

\begin{tabular}{|c|c|c|c|c|}
\hline \multirow{2}{*}{} & \multicolumn{2}{|c|}{ CGM } & \multicolumn{2}{c|}{ DGM } \\
\cline { 2 - 5 } & $\begin{array}{c}\text { Number of } \\
\text { nodes }\end{array}$ & $\begin{array}{c}\text { Number of } \\
\text { degrees of } \\
\text { freedom }\end{array}$ & $\begin{array}{c}\text { Number of } \\
\text { nodes }\end{array}$ & $\begin{array}{c}\text { Number of } \\
\text { degrees of } \\
\text { freedom }\end{array}$ \\
\hline$p=1$ & 1166 & 3498 & 20228 & 242736 \\
\hline$p=2$ & 7982 & 23946 & 50570 & 606840 \\
\hline
\end{tabular}

\section{Conclusions}

CGM is a much more accurate and faster method in calculating displacements. The number of degrees of freedom present in the paper, necessary to determine DGM in the computational process, greatly reduces the effectiveness of the method. Additionally, the slow convergence of the iterative process leading to a steady-state solution makes the situation even worse. DGM reveals its advantages when solving hyperbolic equations (e.g. pure advection) or transient problems with dominant convection, while in the presented case it does not seem to be a good choice.

\section{References}

[1] Studziński, R., Pozorski, Z., \& Błaszczuk, J., (2015). Optimal support system of sandwich panels, Journal of Engineering Mechanics, 141(3), 1-8, 04014133.

[2] Węgrzyn-Skrzypczak, E. (2020). Analysis of the three-dimensional thermoelasticity problem with the use of the continuous Galerkin method. Journal of Applied Mathematics and Computational Mechanics, 19(3), 111-121.

[3] Cockburn, B., Karniadakis, G.E., \& Shu, C.W. (2000). Discontinuous Galerkin Methods. Theory, Computations and Applications, vol. 11 of Lecture Notes in Computational Science and Engineering, Springer, Berlin.

[4] Lew, A., Neff, P., Sulsky, D., \& Ortiz, M. (2004). Optimal BV estimates for a discontinuous Galerkin method for linear elasticity. Applied Mathematics Research Express, 3, 73-106.

[5] Węgrzyn-Skrzypczak, E. (2019). Discontinuous Galerkin method for the three-dimensional problem of thermoelasticity. Journal of Applied Mathematics and Computational Mechanics, 18(4), 115-126.

[6] Riviere, B. (2008). Discontinuous Galerkin methods for solving elliptic and parabolic equations: Theory and implementation. Frontiers in Mathematics 35, SIAM.

[7] Zienkiewicz, O.C. (1977). The Finite Element Method. Mc Graw-Hill, London.

[8] Bathe, K.J. (1982). Finite Element Procedures in Engineering Analysis. Prentice-Hall.

[9] Geuzaine, C., \& Remacle, J.F. (2009). Gmsh: A 3-D finite element mesh generator with built-in pre- and post-processing facilities. Int. J Numer. Meth. Eng., 79, 13091. 